\title{
EL IMPACTO DEL SALARIO MÍNIMO Y DEL EMPLEO INFORMAL SOBRE EL INGRESO SALARIAL EN MÉXICO
}

\author{
Tomás Gómez Rodríguez, ${ }^{a}$ Humberto Ríos Bolívar ${ }^{\mathrm{b}}$ \\ y Adriana Zambrano Reyes ${ }^{\mathrm{a}}$
}

Fecha de recepción: 29 de octubre de 2018. Fecha de aceptación: 6 de mayo de 2019.

http://dx.doi.org/10.22201/iiec.20078951e.2019.199.67516

Resumen. La presente investigación aborda el tema del impacto del salario mínimo y del empleo informal sobre las diferencias del ingreso salarial. Bajo este propósito, se plantea un Modelo de Censura que emplea datos codificados para un subconjunto de la población. La estimación del modelo se realizó con dos técnicas econométricas: Mínimos Cuadrados Ordinarios (MCO) con efectos fijos y el Método Generalizado de Momentos (GMM). En ambos casos, se emplearon microdatos de la ENOE por entidad federativa para el periodo 2005-2014. Los resultados revelan que el salario mínimo amplia la brecha del ingreso salarial, mientras que el sector informal tiene un efecto reductor sobre las diferencias del ingreso salarial.

Palabras clave: salario mínimo; empleo informal; desigualdad del ingreso; panel de datos.

Clasificación JEL: C33; J31; J21; E24; E26.

\section{IMPACTS OF THE MINIMUM WAGE AND INFORMAL EMPLOYMENT ON INCOME EARNINGS IN MEXICO}

\begin{abstract}
The present research examines the issue of how the minimum wage and informal employment impact differences in income earnings. To this end, a Censorship Model is proposed that uses data codified for a subset of the population. Calculations for this model were performed using two econometric methods: Ordinary Least Squares (oLs) and the Generalized Method of Moments (GMM). In both cases, microdata from the National Occupation and Employment Survey where used for each federal entity, covering the period of 2005-2014. The results demonstrate that the minimum wage widens the gap in income earnings, while the informal sector has a reducing effect on differences in income earnings.
\end{abstract}

Key Words: minimum wage; informal employment; income inequality; data panel.

\footnotetext{
${ }^{a}$ Universidad Autónoma del Estado de Hidalgo (UAEH), México; ${ }^{b}$ Instituto Politécnico Nacional (IPN), México. Correos electrónicos: tomas_gomez@uaeh.edu.mx; hriosb@hotmail.com y adriana_zambrano@uaeh.edu.mx, respectivamente.
} 


\section{INTRODUCCIÓN}

En los últimos años ha crecido la preocupación por tratar de recuperar el valor real del salario mínimo. El tema que se discute de manera permanente en el ámbito académico y en los espacios de política pública es sobre los mecanismos para lograr una mejoría en los ingresos de los trabajadores, particularmente, aquella clase donde se encuentran ubicados los de la escala salarial más baja, asegurando una distribución más justa en los ingresos, reduciendo con esto la creciente desigualdad en el ingreso de la población.

Estadísticas recientes, provenientes del Informe Mundial sobre Salarios de la oit (2017), muestran que en promedio en los últimos ańos el crecimiento del salario real en el mundo cayó de manera significativa. De manera más específica, dicho informe señala que el salario real se contrajo 1.3\% en América Latina y el Caribe, siendo México el país con mayor caída (-12\%) durante el periodo 2005-2015, ubicándose por arriba de países como El Salvador (-11\%), Honduras (-6\%), y muy lejos de países como Chile y Brasil (36\%) que registran un aumento en el valor del salario real.

Si bien se sabe que el salario medio de los países no explica el comportamiento de la distribución de los ingresos entre los diferentes grupos de asalariados, existe suficiente evidencia empírica que demuestra que en los últimos años la desigualdad salarial ha aumentado en la mayoría de los países del mundo. Trabajos como los de Maloney (1999), Addison y Blackburn (1999), Lin y Yun (2016) y Neumark et al. (2005) coinciden en que cierto nivel de desigualdad en el ingreso salarial podría ser producto del perfil del trabajador (como nivel de instrucción, edad o antigüedad en el puesto), siendo entonces el perfil del trabajador la variable que podría estar incidiendo en la variación de los ingresos en cada uno de los deciles. Sin embargo, existen otra serie de factores que podrían marcar la diferencia en la variación del nivel de ingreso, entre otros, género del trabajador, tamaño de la empresa y estructura del mercado de trabajo (formal e informal).

No obstante, existe una discusión académica y política sobre el valor del salario real y la demanda de trabajo. Lin y Yun (2016) y Campos-Vázquez et al. (2018a) argumentan que los salarios mínimos crecientes disminuyen las oportunidades de empleo de los trabajadores de bajos ingresos y promueve el empleo informal. Ahí radica el interés de la presente investigación, de analizar el impacto del salario mínimo y del empleo informal sobre las diferencias del ingreso salarial. Una de las principales dificultades para identificar y entender el empleo en el sector informal es la falta de consenso sobre cómo definir y medir las actividades informales. 
Literatura reciente indica que el sector informal es quizá una característica de largo plazo en países emergentes y en desarrollo, en especial en África y América Latina (véase Charmes, 2000). La informalidad representa para estos países una buena parte de la fuerza de trabajo. En este sentido, habría que entender cómo funciona tal aseveración, ya que es esencial para comprender los mercados laborales y la distribución del ingreso. Una de las principales dificultades es tal vez la alta heterogeneidad que caracteriza a dicho sector informal, lo cual dificulta la descripción de su funcionamiento y el papel que juega en la economía. De acuerdo a la visión tradicional propuesta por Fields (1975) y Dickens y Lang (1985), los trabajadores asalariados entran a la informalidad para escapar del desempleo o bien, porque son sacados del sector formal como resultado de la regulación del mercado laboral.

No obstante, algunos trabajos han empezado a cuestionar dicho enfoque, argumentando que una importante parte de los trabajadores informales deciden de manera voluntaria pertenecer al sector informal dada sus preferencias, su dotación de habilidades y los prospectos de ingresos (véase Maloney, 1999). Incluso en modelos recientes del mercado laboral, se sugiere una representación dual del sector informal donde coexisten un segmento voluntario con un segmento racionado (las personas en este segmento están fuera del sector formal debido a los altos salarios y no tienen más opción que incorporarse al sector informal) (véase Funkhouser, 1998; Blunch et al., 2001, y Fields, 2005). Carneiro y Henley (2001) indican que las diferencias en el ingreso se explican por las características no observables de los trabajadores que deciden unirse a cada sector. Wu y Li (2006) demuestran que cerca del $90 \%$ de las diferencias observadas del ingreso mensual entre trabajadores formales e informales en China pueden ser explicadas por características observables.

En el caso de México, 53.8\% de la población se encuentra en el sector informal según datos de la Encuesta Nacional de Ocupación y Empleo (ENOE) elaborado por el INEGi para el periodo 2005-2014. Estudios para México, como los de Samaniego (2006), señalan que el tamaño del sector informal es un factor subyacente que explica temas como la desigualdad del ingreso, la pobreza y la ineficiencia del mercado laboral, además, el tamaño del sector informal podría funcionar como válvula de escape para las personas cuando disminuye su ingreso o cuando pierden su empleo. Como resultado de la crisis global, en 2009 se observó una disminución del ingreso para todos los deciles; en ese mismo año se registró un aumento en el tamaño del sector informal. Lo anterior parece sugerir que el sector informal podría tener un efecto reductor sobre la desigualdad del ingreso. 
Como hipótesis de partida de esta investigación, se plantea que el salario mínimo tendrá un efecto negativo sobre las diferencias del ingreso salarial, mientras que el empleo informal tendrá un efecto reductor en las diferencias del ingreso salarial. Para el análisis de las dos proposiciones anteriores se parte del Modelo de Censura propuesto por Autor et al. (2016). Este modelo se ha empleado sobre todo para examinar la primera relación antes mencionada; sin embargo, como se señala en el trabajo de Bosch y Manacorda (2010) existen otros factores como el efecto del comercio internacional, los factores macroeconómicos y los impactos del tipo de cambio, los cuales pueden ayudar a explicar las diferencias del ingreso salarial. En este sentido, se busca profundizar en las posibles explicaciones de las diferencias del ingreso salarial, incorporando al análisis la variable tamaño del sector informal para el caso de México.

Cabe señalar que la presente investigación está orientada a la realización de un análisis econométrico empleando una muestra de 32 entidades federativas para el período 2005-2014. El análisis intenta contribuir a la discusión respecto a la relevancia del valor del salario mínimo en la desigualdad del ingreso en México y verificar, si persiste en el tiempo, la relación positiva encontrada en Bosch y Manacorda (2010). Al cambiar la base de datos por una muestra de las 32 entidades federativas en lugar de municipios urbanos -por cuestiones de variabilidad de ingresos en los distintos deciles-, se requiere de una base de datos más heterogénea, razón por la cual se optó por utilizar información por entidad federativa.

Algunas otras diferencias sustanciales con la propuesta Bosch y Manacorda (2010) son que mientras en su trabajo se emplea el Método de Variables Instrumentales en la estimación del modelo, en la presente investigación se estima en primera instancia el modelo de panel mediante el Método de Mínimos Cuadrados Ordinarios (MCO) con efectos fijos y en una segunda instancia se estima el modelo a través del Método Generalizado de Momentos (MGM) como prueba de robustez.

La estructura del trabajo es la siguiente: en la sección 2 se presenta una discusión de la literatura existente sobre el tema del salario mínimo y su impacto sobre las diferencias del ingreso, asimismo, se realiza una revisión de la literatura sobre el tema del empleo informal; en la sección 3, se especifica el modelo que se utilizará en la investigación; en la sección 4, se discuten los datos; finalmente, en las secciones 5 y 6 se presentan los principales resultados y las conclusiones, respectivamente. 


\section{REVISIÓN DE LA LITERATURA}

Existe una amplia literatura sobre el tema del salario mínimo y su relación con la desigualdad en el ingreso. Addison y Blackburn (1999) analizan el efecto de los salarios mínimos sobre los niveles de pobreza en México, utilizan un modelo de panel con información por entidad federativa, y encuentra que el salario mínimo tiene un efecto significativo en la reducción de la pobreza, así como en la población de adolescentes que abandonan la preparatoria.

En tanto que Neumark et al. (2005) utilizan un método no paramétrico para estimar el efecto del salario mínimo en la desigualdad del ingreso para el caso de Estados Unidos de América. Los resultados muestran que, para el grupo inferior de la distribución de ingreso, el salario mínimo podría aumentar la desigualdad. Por otro lado, trabajos para el caso de México como los de Bosch y Manacorda (2010) y Duval-Hernández et al. (2016), coinciden en que el salario mínimo explica buena parte de la inequidad del ingreso. Ambos autores encuentran que, en el nivel inferior de la distribución del ingreso, la mayor parte de la desigualdad del ingreso se puede atribuir a la rápida disminución en el valor real de los salarios mínimos. También encuentran que los cambios en el panel son convergentes y que dicha convergencia es producida más por cambios en el mercado laboral que por el perfil de los trabajadores (escolaridad, edad y género).

Desde otra perspectiva, donde se discute las consecuencias en el mercado laboral ante un aumento en el salario mínimo, Moreno-Brid et al. (2014) destacan que el salario mínimo en México ha sufrido un agudo deterioro durante varias décadas debido a la inflación y que es necesario desvincularlo como referencia para transacciones fuera del ámbito laboral. Campos-Vázquez et al. (2018b) presentan nuevas metodologías para determinar los ingresos de los percentiles más altos de la distribución del ingreso. Para estimar el ingreso de los individuos en el decil más alto de la distribución del ingreso en México desde 1992, Campos-Vázquez et al. (2018b) emplean información de las encuestas de los hogares, para ajustar la mala representación de las personas con ingresos más altos usando datos de las cuentas nacionales. Se estima el ingreso de las personas más ricas empleando interpolaciones de la distribución de Pareto y encuentran que la proporción del ingreso del percentil más alto es cercano al $25 \%$.

En otro trabajo más reciente, Campos-Vázquez et al. (2018a) estudian la relación entre el aumento del salario mínimo y el empleo en México. Para ello, se homologa el salario mínimo en dos zonas del país a finales de 2012. Los resultados indican que, en promedio, en las zonas en donde aumenta el salario 
mínimo, también disminuye la probabilidad de ser trabajador informal. Por su parte, Bouchot (2018), evalúa de manera empírica las implicaciones de un aumento del salario mínimo sobre la distribución del ingreso, el empleo y el empleo informal. Utiliza como un experimento natural la armonización parcial de los salarios mínimos regionales en 2012. A través del Método de Estimación de Diferencias en Diferencias, los resultados sugieren que no se encuentra evidencia de un efecto adverso sobre el empleo. En lugar de eso, se encuentran efectos positivos sobre los salarios reales por hora, empleo y ocupación en el sector formal.

Existe una literatura limitada sobre las diferencias del ingreso y su relación con el empleo en el sector informal y formal. Trabajos como los de Tansel y Kan (2016) sugieren que, ante un aumento en el salario mínimo en el desempleo formal, el sector informal es una válvula de escape, pero los trabajadores informales siempre estarán en desventaja por las condiciones precarias a que se enfrentan, además, encuentran que se agudiza la discriminación de género entre los trabajadores del sector informal. Estos resultados coinciden con la teoría tradicional de que los trabajadores en el sector formal reciben salarios más altos que los trabajadores que pasan al sector informal, quienes padecen mayores condiciones de precariedad laboral.

De manera más general, existen estudios para otros países sobre diferencias en el ingreso, por ejemplo, Bargain y Kwenda (2010) estudian los casos de Brasil, México y Sudáfrica. Los resultados de este trabajo confirman que los trabajadores del sector informal reciben un salario más bajo debido, principalmente, al bajo perfil y las habilidades que presentan estos trabajadores. De igual forma, Staneva y Arabsheibani (2014), en el análisis para el caso de Tayikistán encuentran que las diferencias salariales observadas son atribuidas al bajo perfil y a las habilidades de los trabajadores del sector informal. Por su parte Rand y Torm (2012) en un estudio sobre Vietnam, analiza el sector informal, y sus resultados confirman tanto la existencia de una brecha del ingreso salarial entre el sector formal e informal, como que los salarios promedios son de 10 a $20 \%$ más altos en las empresas formales con respecto a las empresas informales.

En otro estudio, realizado para China, Zuo (2013) examina también las diferencias del ingreso entre trabajadores formales e informales en las zonas urbanas del país, donde los resultados revelan que sólo 33\% del diferencial salarial observado puede ser explicado por el perfil del trabajador, y $67 \%$ es atribuible al efecto de segmentación. 


\section{IDENTIFICACIÓN Y ESPECIFICACIÓN DEL MODELO}

Con el propósito de analizar el efecto del salario mínimo en las diferencias del ingreso salarial, se tomaron los trabajos de Lee (1999) y de Autor et al. (2016). Lee (1999) modela la relación entre la variación en el efecto del salario mínimo y la distribución del salario, identificando el crecimiento promedio de la desigualdad en el ingreso en función del salario mínimo, y establece que la relación teórica de dichas variables puede darse por truncamiento, censura o por derrame.

Para analizar la interacción entre salario mínimo y distribución del ingreso salarial, se utiliza el Modelo de Censura, cuya principal característica radica en la flexibilidad para incluir variables latentes (variables que no son directamente observables). Es decir, el modelo parte de una variable latente, pero de la cual se puede tener información censurada. El modelo supone además un indicador $d_{i}$ mediante el cual se describe el proceso de censura, siendo $d_{i}=0$ una observación sin censura y $d_{i}=1$ y la ecuación de censura está representada por:

$x_{i}^{c e n}=\left(1-d_{i}\right) x_{i}+d_{i} \xi$

Donde $x_{i}^{\text {cen }}$ es la versión censurada de $x_{i}$ y $\xi$ representa el valor de censura. La probabilidad de censura es denotada como $p=\operatorname{Pr}\{d=1\}$ y se asume $0<p<1$.

Por otra parte, el proceso de censura $d_{i}$ puede ser bastante general, por lo que se especifica un proceso de censura con un solo valor para algún límite. Por ejemplo, un proceso de censura de límite superior involucra observar $x_{i}$ sólo cuando es menor al límite $\xi$, esto es:

$d_{i}=1\left[x_{i}>\xi\right]$

siendo $\xi$ el límite del valor de censura. Una vez establecida la ecuación que representa el Modelo de Censura, el siguiente paso es establecer la relación entre el salario mínimo y la distribución del ingreso salarial. Dicha relación está dada por la diferencia entre el percentil objetivo $w_{m t}^{q}$ y el percentil usado como medida de centralidad $\boldsymbol{w}_{m t}^{p}$ expresada en la siguiente ecuación: $\mathcal{w}_{m t}^{q}-\boldsymbol{w}_{m t}^{p}=$ diferencias del ingreso salarial 
donde $w_{m t}^{q}$ es el q_ésimo percentil de la distribución logarítmica del ingreso salarial en el estado $m$ y en el tiempo $t$. Las diferencias del ingreso salarial se pueden considerar como una medida de desigualdad, que considera la distancia entre el ingreso en el percentil objetivo y una medida de centralidad; valores altos de esta variable indican mayor desigualdad, mientras que valores bajos indican menor desigualdad. Al suponer que existe un percentil $p$ suficiente alto, tal que los salarios iguales a este percentil o a uno más alto no se vean afectados por el salario mínimo. Por tanto $w_{m t}^{p}$ es el $p \_e ́ s i m o$ percentil que se establece como medida de centralidad en el estado $m$ y en el tiempo $t$. Por otro lado, para medir el efecto de la variable salario mínimo sobre las diferencias del ingreso, se define al salario mínimo efectivo $(W O)$ como la diferencia entre el salario mínimo y una medida de centralidad; se expresa de la siguiente manera:

$$
M W_{m t}-w_{m t}^{p}=W O
$$

Donde $M W_{m t}$ es el logaritmo del salario mínimo nominal en el estado $m$ en el tiempo $t$. Una vez definidas las variables se replantea el Modelo de Censura. Este modelo asume que todos los trabajadores que obtienen un salario latente por debajo del salario mínimo obtienen el salario mínimo y todos lo que están por encima no se ven afectados. El Modelo de Censura implica que el diferencial del ingreso salarial del logaritmo $q$ a $p$ se expresar como:

$$
\mathcal{W}_{m t}^{q}-\mathcal{W}_{m t}^{p}=\mathcal{W}_{m t}^{* q}-\mathcal{W}_{m t}^{*_{p}} \text { si } \mathcal{W}_{m t}^{* p} \geq M W_{m t}
$$

Donde $w_{m t}^{*_{q}}-w_{m t}^{*_{p}}$ es la diferencia salarial latente, donde $w_{m t}^{*_{q}}$ es el $q \_e ́ s i m o$ percentil latente del estado $m$ en el tiempo $t$ y $w_{m t}^{* p}$ es el $p \_e ́ s i m o$ percentil latente del estado $m$ en el tiempo $t$. Lo anterior significa que el diferencial del ingreso observado es igual al diferencial del ingreso latente si $w_{m t}^{* q}$ es mayor o igual a $M W_{m t}$.

$$
\mathcal{w}_{m t}^{q}-\mathcal{w}_{m t}^{p}=W O \text { si } w_{m t}^{* q}<M W_{m t}
$$

Por otro lado, si $w_{m t}^{* q}$ es menor a $M W_{m t}$ entonces el diferencial del ingreso observado es igual al salario mínimo efectivo. Bajo estas condiciones se fija el valor de la medida de centralidad $p$. Lee (1999) y Autor et al. (2016) establecen un nivel de 50, significa que el percentil 50 sería la medida de centralidad para el caso de Estados Unidos de América. La elección del percentil 70 como medida de centralidad proviene de la hipótesis de que existen efectos de derrame. Según el trabajo de Bosch y Manacorda (2010), esta medida de cen- 
tralidad puede ser un supuesto razonable para México, ya que existe evidencia de desbordes del salario mínimo en los percentiles por encima del percentil 50. Una vez determinada la medida de centralidad, la diferencia del ingreso salarial está determinada por:

$w_{m t}^{q}-w_{m t}^{70}=$ diferencias del ingreso

Asimismo, la ecuación del salario mínimo efectivo una vez que se incluye la medida de centralidad es la siguiente:

$$
M W_{m t}-w_{m t}^{70}=W O
$$

Por otra parte, para poder hacer la estimación se requiere parametrizar el modelo, para lo cual se debe expresar la dispersión salarial como una función del diferencial del salario latente más el efecto del salario mínimo.

diferencias del ingreso $=w_{m t}^{* q}-w_{m t}^{* 70}+W O$

De donde el efecto del salario se expresa como una función cuadrática del salario mínimo real, es decir:

$W O=W O+W O^{2}$

Finalmente, la dispersión salarial latente se expresa mediante:

$w_{m t}^{* q}-w_{m t}^{* 70}=\alpha_{m}^{q}+X_{m t}^{\prime}+U_{m t}$

Donde $\alpha_{m}^{q}$ son características específicas de cada estado y $X_{m t}^{\prime}$ es un vector adicional de covariables específicas de cada estado y $U_{m t}$ es el término de error.

De lo anterior, se obtiene el nuevo modelo de regresión el cual está asociado al modelo de censura, este se expresa de la siguiente manera:

diferencias del ingreso $=\alpha_{m}^{q}+\alpha_{t}^{q}+\beta_{1} W O+B_{2} W O^{2}+X_{m t}^{\prime}+u_{m t}$

Donde $u_{m t}$ es el término de error. La ecuación (12) representa una alternativa simple paramétrica al modelo de cesura de la ecuación (11) y es la base de nuestro análisis empírico. Cabe señalar que en el vector $X_{m t}^{\prime}$ se incluyen las variables de control: número de horas trabajadas $(H W)$, tasa de asalariados $(T A)$ y tasa de informalidad (TIL2). Se espera que el signo de la variable $H W$ sea negativo, debido a que los trabajadores pueden aumentar el número de horas para alcanzar un mayor ingreso y con esto disminuir las diferencias del ingreso. La 
variable $T A$ mide el porcentaje de trabajadores asalariados, se espera que tenga signo positivo, ya que los salarios en el sector formal son más altos. Por último, TIL2 es la variable empleada para medir el efecto del sector informal sobre la distribución del ingreso, se espera que aumente las diferencias del ingreso.

Para realizar la estimación de la ecuación (12), se calcularon los percentiles del ingreso salarial de las 32 entidades federativas que componen la República mexicana, utilizando datos del cuarto trimestre de cada año, correspondientes al periodo 2000-2014. De una muestra total de 390828 individuos por trimestre, se seleccionaron a los individuos que reportaron haber recibido un ingreso, lo que redujo la muestra a 118 400. Dando como resultado una muestra promedio de 3700 individuos por entidad federativa.

Aunque no se impuso ninguna restricción a priori en el valor de los parámetros, el modelo garantiza, que por lo menos en un rango de variación definido de $W O$, el diferencial observado del ingreso salarial tiende a $W O$ cuando $W O$ crece, y tiende a $w_{m t}^{*_{q}}-w_{m t}^{*_{p}}$ cuando $W O$ disminuye, esto es consistente con el modelo de censura en la ecuación (11).

Una de las ventajas de utilizar la ecuación (12), es que permite detectar asimetrías en el salario mínimo en los percentiles más altos de la distribución. Para el análisis econométrico, se utiliza información en forma de panel y se estima la ecuación (12) mediante los métodos de MCO y de efectos fijos (véanse los resultados en el cuadro 2).

Uno de los problemas principales en la estimación de la ecuación (12) surge de la posibilidad de correlaciones positivas espurias entre el diferencial del ingreso observado y el salario mínimo efectivo. Este problema tiene su origen en los errores de muestreo y del hecho de que el percentil 70 está en ambos lados de la ecuación. A esta situación hacen referencia Autor et al. (2016) y la denominan "problema de división de sesgo". Existe la posibilidad de que al estimar el modelo mediante MCO y efectos fijos, no exista la relación entre estas dos medidas o que dicha relación sea muy pequeña, pero estadísticamente significativa; este tipo de interacción puede deberse a sesgo en la información. La fuente de sesgo debe tomarse en cuenta para comprobar la robustez del modelo y evitar resultados engañosos. Para resolver este problema, una posible solución es estimar la ecuación (13) usando GMM. Como condición para aplicar el método GMM se aplica el logaritmo en el cálculo de las diferencias del ingreso salarial como en Kambayashi et al. (2013). Por lo que la ecuación a estimar es la siguiente:

diferencias del ingreso $=\beta_{0}+\beta_{1} \ln W O_{m t}+\beta_{2} \ln W O 2_{m t}+\beta_{3} H W_{m t}+$

$$
\beta_{4} T A_{m t}+\beta_{5} T I L 2_{m t}+u_{m t}
$$


La justificación de esta estimación es que método GMM es más eficiente y flexible debido a que se puede obtener más información usando variables de control y rezagos de las variables como instrumentos de estimación. Otra fortaleza de la técnica GMM es que permite realizar pruebas más exhaustivas, sobre todo para los percentiles 80 y 90 en donde se espera que el salario mínimo no tenga un impacto en los percentiles más altos. Los resultados de la estimación se muestran en el cuadro 3.

\section{ANÁLISIS DESCRIPTIVO DE LA INFORMACIÓN ESTADÍSTICA}

Para analizar la interacción entre salario mínimo y las diferencias en el ingreso en México, se usan micro datos de la ENOE para el periodo 2005-2014. La ENOE es la consolidación y fusión de la Encuesta Nacional de Empleo Urbano (ENEU) y la Encuesta Nacional de Empleo (ENE) que, por más de 20 años, proporcionaron información de la población ocupada y desocupada, similar a la encuesta de población actual de Estados Unidos de América.

De igual manera, para analizar la relación entre empleo informal y diferencias del ingreso, se utilizó información estadística de la ENOE, ya que es la encuesta oficial del mercado laboral y que tiene disponible información desde finales de los años ochenta del siglo xx. Las unidades de la muestra son las entidades federativas, lo que permite estructurar dicha información estadística como un panel compuesto por 32 bloques de información para México.

En el cuadro 1 se presenta una breve descripción estadística de la información proporcionada por la ENOE sobre las variables independientes del modelo. Un aspecto que sobresale en la información proporcionada por el cuadro 1, es que el nivel de eficiencia de los salarios siempre presenta un signo negativo.

Cuadro 1. Estadísticas descriptivas de las variables independientes del modelo

\begin{tabular}{llccrr}
\hline & Abreviatura & Media & Máximo & Mínimo & Desviación estándar \\
\hline Número de horas trabajadas & HW & 42.4 & 46.3 & 37.9 & 1.6 \\
Tasa de informalidad & TIL2 & 53.8 & 74.1 & 32.9 & 10.11 \\
Tasa de asalariados & TA & 61.9 & 78.7 & 35.9 & 9.15 \\
Salario eficiente & WO & -1.19 & -0.81 & -1.53 & 0.13 \\
\hline
\end{tabular}

Fuente: elaboración propia con datos de INEGI-ENOE (2018). 
En tanto que la gráfica 1 tiene el propósito de examinar la distribución de los ingresos obtenidos por nivel de percentil. En ella se destaca que en el percentil superior de la distribución, el $0.1 \%$ de la población con mayores ingresos, tiene una remuneración de MXN\$60 547.2; mientras que el ingreso promedio del 1\% de la población con mayores ingresos es de MXN\$24 423.7. Por otro lado, al calcular un salario mínimo mensual promedio para el periodo 2005-2014 del estudio, se obtiene MXN\$1 648.3; mientras que el ingreso promedio del percentil 10 es MXN\$1 379.8, lo que significa que el ingreso promedio del décimo percentil está por debajo del salario mínimo promedio y la diferencia es de MXN\$268.58. Como se puede observar, la gráfica 1 muestra la variabilidad en la distribución de ingresos en los distintos deciles.

La gráfica 2 muestra que, si bien existe poca variación del salario real en el periodo de estudio, la variación de los ingresos entre deciles es más observable. La gráfica muestra una tasa de crecimiento del ingreso en los percentiles del 10 al 90 para el periodo 2006-2014. En 2009 todos los percentiles muestran una tasa de crecimiento negativo seguida de una recuperación moderada en 2010. El percentil 10 es el más afectado con una tasa de crecimiento de $-6.3 \%$. En 2011 los percentiles 10 y 20 registran una tasa de crecimiento de -3.65 y $-0.74 \%$, respectivamente. Finalmente, la tasa de crecimiento del ingreso del percentil 10 más alto es de $8.48 \%$ y se registra en 2012 . La segunda mayor tasa de crecimiento se registra en 2006 con un valor de $7.41 \%$.

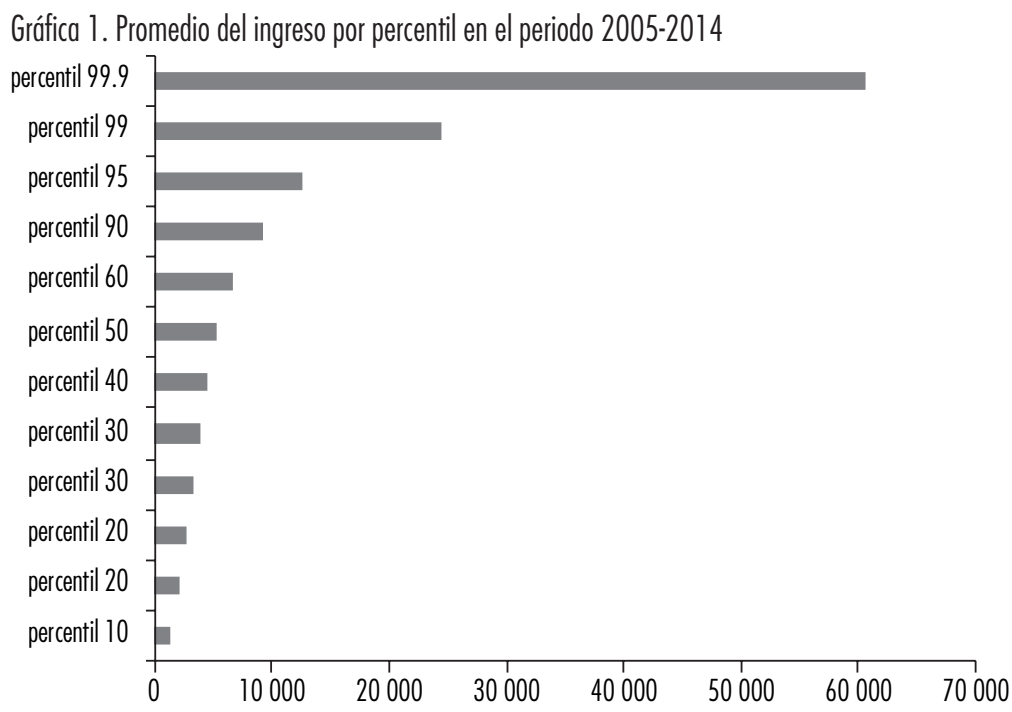

Fuente: elaboración propia con datos de INEGI-ENOE (2018). 


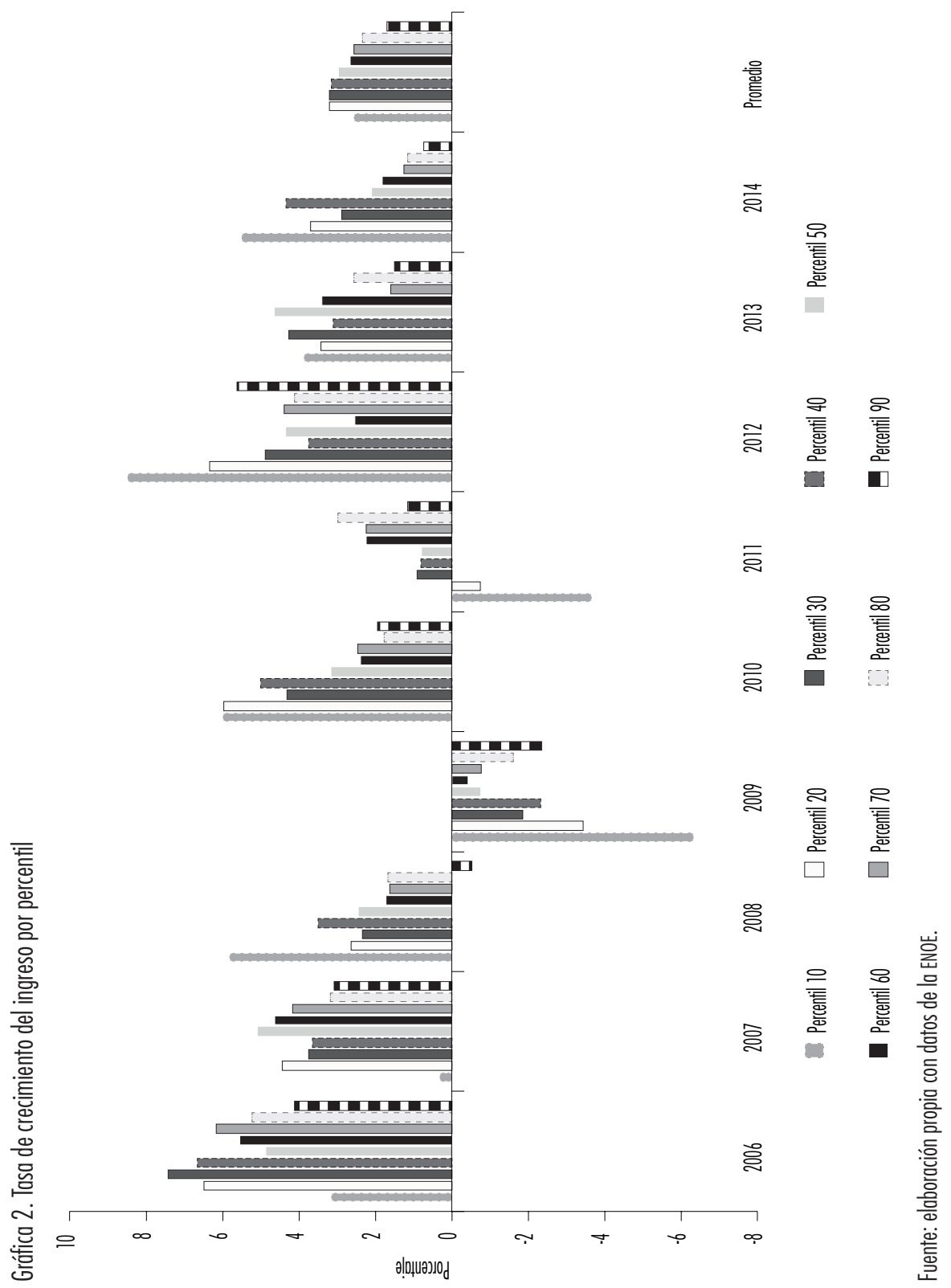


Las gráficas 3, 4 y 5, muestran la variación de crecimiento de las diferencias del ingreso, de la tasa de crecimiento del salario eficiente y de la tasa de crecimiento del empleo informal. En la gráfica 3, se observa una disminución de la tasa de crecimiento de las diferencias del ingreso para los años 2008, 2009 y 2010. De igual manera, como se muestra en la gráfica 4, dicha disminución coincide con tasas negativas del crecimiento del salario y de manera inversa, existe un crecimiento del empleo informal en el mismo periodo como se muestra en la gráfica 5 . Esto sugiere que el empleo informal podría reducir las diferencias del ingreso al servir como una alternativa a los bajos ingresos del sector formal.

Gráfica 3. Panel sobre la tasa de crecimiento de las diferencias del ingreso, 2006-2014

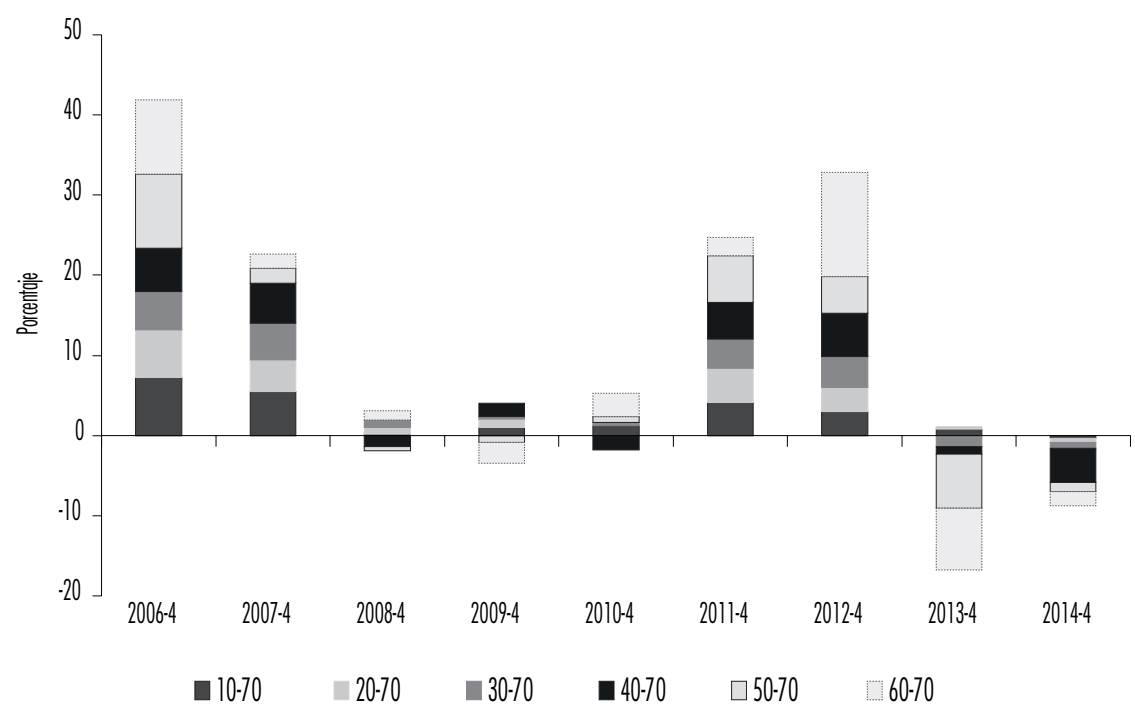

Fuente: elaboración propia con datos de la ENOE. 
Gráfica 4. Panel sobre la tasa de crecimiento del salario eficiente, 2006-2014

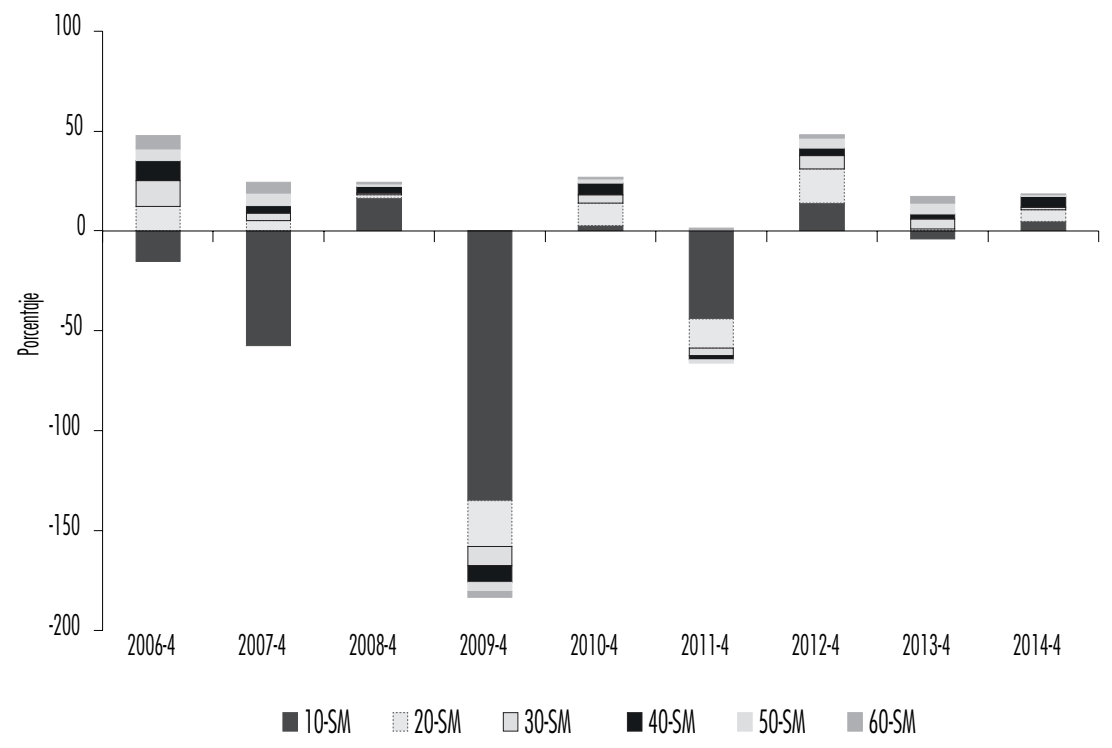

Fuente: elaboración propia con datos de la ENOE.

Gráfica 5. Panel sobre la tasa de crecimiento del empleo informal, 2006-2014

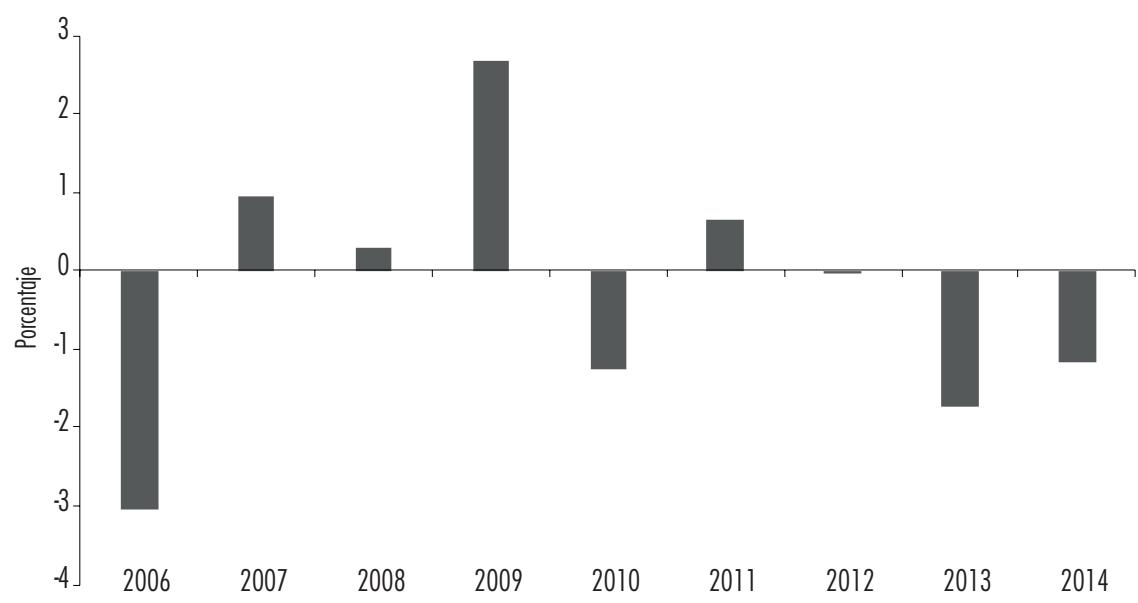

Fuente: elaboración propia con datos de la ENOE. 


\section{RESULTADOS}

El cuadro 2 muestra los resultados de la estimación empleando el MCO con efectos fijos para los primeros cuatro deciles. Los resultados de la prueba $\mathrm{F}$ refieren que, en conjunto, todos los parámetros en los modelos son estadísticamente significativos (ES). Por su parte, la prueba Durbin-Watson no encuentra evidencia de la existencia de autocorrelación de los errores.

Cabe señalar que cada entrada en los cuadros 2 y 3 se refiere al coeficiente para cada variable de la regresión entre cada diferencia del ingreso salarial con respecto al séptimo decil, que juega el papel de pivote, por estado y año. El método de estimación es el de MCO con efectos fijos usando datos de la ENOE.

Cuadro 2. Impacto del salario mínimo en los diferenciales del ingreso salarial deciles 1-4, usando MCO

\begin{tabular}{|c|c|c|c|c|c|}
\hline Variable & Parámetro & Diferencia 10-70 & Diferencia 20-70 & Diferencia 30-70 & Diferencia 40-70 \\
\hline \multirow[t]{2}{*}{ Intercepto } & $\beta_{0}$ & -0.863182 & $-0.971970^{\star \star \star}$ & $-1.057584^{\star \star \star}$ & $-0.632936^{*}$ \\
\hline & & $(.2134)$ & $(.0314)$ & $(.0050)$ & $(.0651)$ \\
\hline \multirow[t]{2}{*}{ wo } & $\beta_{1}$ & $0.392261^{* \star *}$ & $0.530740^{\star \star \star}$ & $0.388220^{\star \star \star \star}$ & $0.459841^{\star \star \star}$ \\
\hline & & $(.0001)$ & $(.0000)$ & $(.0000)$ & $(.0000)$ \\
\hline \multirow[t]{2}{*}{ W02 } & $\beta_{2}$ & 0.003492 & $0.112114^{\star \star \star}$ & $0.076911^{\star \star}$ & $0.085185^{\star \star \star *}$ \\
\hline & & $(.9411)$ & $(.0003)$ & $(.0028)$ & $(.0003)$ \\
\hline \multirow[t]{2}{*}{ HW } & $\beta_{3}$ & $0.015315^{\star}$ & $0.010288^{*}$ & $0.009612^{\star \star}$ & 0.007122 \\
\hline & & $(.0820)$ & $(.0720)$ & $(.0028)$ & (.1014) \\
\hline \multirow[t]{2}{*}{ TA } & $\beta_{4}$ & -0.000953 & 0.002183 & -0.001156 & -0.000506 \\
\hline & & $(.6986)$ & $(.1725)$ & $(.3845)$ & $(.6769)$ \\
\hline \multirow[t]{2}{*}{ TIL2 } & $\beta_{5}$ & $-0.009656^{\star \star \star}$ & $-0.006187^{\star \star \star}$ & $-0.005751^{\star \star \star}$ & $-0.003827^{\star \star \star}$ \\
\hline & & $(.0014)$ & $(.0016)$ & $(.0004)$ & $(.0102)$ \\
\hline $\begin{array}{l}\text { Coeficiente de } \\
\text { determinación }\end{array}$ & $R^{2}$ & .92 & .92 & .86 & .82 \\
\hline Estadístico F & $\mathrm{F}$ & 88.8 & 80.3 & 43.6 & 32.6 \\
\hline $\begin{array}{l}\text { Estadístico } \\
\text { Durbin-Watson }\end{array}$ & DW & 1.87 & 1.77 & 1.46 & 1.69 \\
\hline
\end{tabular}

Nota: ${ }^{\star \star \star}$ Es al $1 \%,{ }^{\star \star}$ Es al $5 \%$ y Es al $10 \%$.

Fuente: elaboración propia con datos de la ENOE. Los valores de probabilidad están entre paréntesis. 
Cuadro 3. Impacto del salario mínimo en los diferenciales del ingreso salarial, deciles 5-9, usando MCO

\begin{tabular}{|c|c|c|c|c|c|}
\hline Variable & Parámetro & Diferencia 50-70 & Diferencia 60-70 & Diferencia 80-70 & Diferencia 90-70 \\
\hline \multirow[t]{2}{*}{ Intercepto } & $\beta_{0}$ & -0.059965 & $0.536244^{\star}$ & $0.912822^{*}$ & $1.836376^{\text {*** }}$ \\
\hline & & $(.8429)$ & $(.0617)$ & $(.0063)$ & $(.0000)$ \\
\hline \multirow[t]{2}{*}{ wo } & $\beta_{1}$ & $0.459911^{\star \star \star}$ & 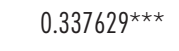 & $0.275623^{\star \star \star}$ & 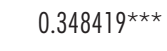 \\
\hline & & $(.0000)$ & $(.0000)$ & $(.0000)$ & $(.0000)$ \\
\hline \multirow[t]{2}{*}{ W02 } & $\beta_{2}$ & $0.076945^{\star \star \star}$ & $0.063931^{\star \star *}$ & $0.044666^{\star *}$ & $0.080556^{\text {*** }}$ \\
\hline & & $(.0002)$ & $(.0012)$ & $(.0495)$ & $(.0066)$ \\
\hline \multirow[t]{2}{*}{ HW } & $\beta_{3}$ & 0.003858 & -.0000828 & 0.001131 & -0.000303 \\
\hline & & $(.3149)$ & $(.9818)$ & (.7882) & $(.9559)$ \\
\hline \multirow[t]{2}{*}{ TA } & $\beta_{4}$ & $-0.002074^{\star \star}$ & $-0.002584^{\star \star \star}$ & $-0.002632^{\star \star}$ & $-0.004042^{\star \star \star}$ \\
\hline & & $(.0544)$ & $(.0114)$ & $(.0262)$ & $(.0087)$ \\
\hline \multirow[t]{2}{*}{ TIL2 } & $\beta_{5}$ & $-0.002929^{\star \star}$ & $-0.002246^{*}$ & $-0.002517^{\star}$ & $-0.003576^{\star \star}$ \\
\hline & & $(.0261)$ & $(.0707)$ & $(.0809)$ & $(.0564)$ \\
\hline $\begin{array}{l}\text { Coeficiente de } \\
\text { determinación }\end{array}$ & $R^{2}$ & .73 & .45 & .4 & .67 \\
\hline Estadístico F & $\mathrm{F}$ & 18.97 & 5.87 & 4.7 & 14.42 \\
\hline $\begin{array}{l}\text { Estadístico } \\
\text { Durbin-Watson }\end{array}$ & DW & 1.69 & 1.62 & 1.9 & 1.78 \\
\hline
\end{tabular}

Nota: ${ }^{\star \star \star}$ Es al $1 \%,{ }^{\star \star}$ Es al $5 \%$ y Es al $10 \%$.

Fuente: elaboración propia con datos de la ENOE. Los valores de probabilidad están entre paréntesis.

La información de los cuadros 2 y 3 muestran que la variable salario eficiente tiene signo positivo y es al $1 \%$ en todas las diferencias del ingreso. Por tanto, un incremento de $10 \%$ en la variable $W O$ significa un incremento de $3.9 \%$, $5.3 \%, 3.8 \%, 4.5 \%, 4.5 \%, 3.3 \%, 2.7 \%$ y $3.4 \%$ para las diferencias del ingreso 10-70, 20-70, 30-70, 40-70, 50-70, 60-70, 80-70 y 90-70, respectivamente.

De igual manera el término cuadrático del salario eficiente tiene signo positivo y es es para todas las diferencias del ingreso con la excepción de la diferencia 10-70. $H W$ es significativa sólo para las tres primeras diferencias del ingreso y su signo es positivo el cual no es el esperado. La variable $T A$ es ES para las diferencias 50-70, 60-70, 80-70 y 90-70 y presenta un signo negativo el cual no es el esperado. La variable TIL2 es ES y exhibe signo negativo para todas las diferencias. Un aumento de $10 \%$ en la variable TIL2 significa una reducción de las diferencias del ingreso del .09\%, .06\%, .05\%, .03\%, .02\%, 
$.02 \%, .02 \%$ y $.03 \%$ para las diferencias del ingreso $10-70,20-70,30-70,40-$ 70, 50-70, 60-70, 80-70 y 90-70, respectivamente.

Se encuentra que el mayor efecto del salario efectivo se observa en los tres primeros percentiles, para el resto de los percentiles se observa que sigue existiendo un efecto positivo de menor magnitud. En otras palabras, los resultados sugieren la existencia de una relación positiva entre salario mínimo y las diferencias del ingreso salarial. Sin embargo, estos resultados pueden verse afectados por el problema de división de sesgo al cual hace referencia Autor et al. (2016). Por lo que se realizó la estimación usando GMM como prueba de robustez de los resultados.

Los resultados de la estimación econométrica utilizando el método GMM se pueden observar en el cuadro 4. Estos reflejan que para la variable $W O$ los coeficientes del parámetro para todas las diferencias del ingreso salarial son positivas, esto significa que un aumento de $10 \%$ del salario incrementa las diferencias del ingreso salarial en $2.4 \%$ en el caso de la diferencia $10-70$. Siendo el valor más alto para el coeficiente de determinación de la variable $W O$ $3.2 \%$ en la diferencia del ingreso 30-70. Mientras que el valor más bajo del coeficiente de determinación de la variable $W O$ es .9\% en la diferencia 90-70. Por otro lado, la variable $W O$ es Es al $1 \%$ en todas las diferencias del ingreso salarial con la excepción de las diferencias $80-70$ y $90-70$. Se advierte una disminución de los coeficientes de $W O$, después de los tres primeros deciles, lo cual es consistente con el efecto esperado, ya que se asume que cuando nos acercamos al decil 70 el efecto del salario mínimo sobre las diferencias del ingreso debe desaparecer.

Los coeficientes para la variable $W O 2$ no son significativos estadísticamente con la excepción de la diferencia 90-70. La variable $H W$ es estadísticamente significativa al 5\% en las diferencias 20-70, 30-70, y Es al 10\% en las diferencias 40-70, 50-70 y 60-70. Todas las diferencias antes mencionadas tienen coeficientes con signo negativo lo que implica que un aumento en esta variable significa una disminución de las diferencias del ingreso salarial. En el caso de las diferencias $20-70$ y 30-70 un aumento de $10 \%$ en esta variable significa una reducción de .09\% y $.05 \%$ en las diferencias del ingreso salarial siendo estos los valores más altos.

El porcentaje de trabajadores asalariados está medido por la variable $T A$ la cual es Es en todas las diferencias salvo en la diferencia 80-70. Para la diferencia 10-70 la variable es Es al 10\%, por otro lado, las diferencias 20-70, 30-70, $40-70,50-70$ y $60-70$ son Es al $1 \%$. Un aumento del $10 \%$ en la variable $T A$ para la diferencia $10-70$ produce un aumento de $.04 \%$ en la diferencia del ingreso salarial. Para las diferencias 20-70, 30-70, 40-70, 50-70 y 60-70 un 
Cuadro 4. Impacto del salario mínimo en los diferenciales del ingreso, usando el modelo GMM, para los deciles 1-4

\begin{tabular}{|c|c|c|c|c|c|}
\hline Variable & Parámetro & Diferencia 10-70 & Diferencia 20-70 & Diferencia 30-70 & Diferencia 40-70 \\
\hline \multirow[t]{2}{*}{ WO } & $\beta_{1}$ & $0.242819^{\star \star}$ & $0.228573^{\star \star \star}$ & $0.329912^{\star \star \star}$ & $0.215500^{\star * *}$ \\
\hline & & $(.0468)$ & $(.0012)$ & $(.0000)$ & $(.0000)$ \\
\hline \multirow[t]{2}{*}{ W02 } & $\beta_{2}$ & -0.104078 & -0.045369 & 0.015301 & -0.000536 \\
\hline & & $(.3260)$ & $(.4574)$ & $(.7251)$ & (.9883) \\
\hline \multirow[t]{2}{*}{ HW } & $\beta_{3}$ & -0.008441 & $-0.009442^{\star \star}$ & $-0.005906^{\star \star}$ & $-0.007872^{\star \star \star}$ \\
\hline & & $(.1981)$ & $(.0128)$ & $(.0289)$ & $(.0006)$ \\
\hline \multirow[t]{2}{*}{ TA } & $\beta_{4}$ & $0.004331^{*}$ & $0.005360^{\star \star \star}$ & $0.003723^{\star \star \star}$ & $0.004133^{\star * \star}$ \\
\hline & & $(.0599)$ & $(.0001)$ & $(.0001)$ & $(.0000)$ \\
\hline \multirow[t]{2}{*}{ TIL2 } & $\beta_{5}$ & $-0.016764^{\star \star \star}$ & $-0.009481^{\star \star \star}$ & $-0.004974^{\star \star \star}$ & $-0.002786^{\star \star \star}$ \\
\hline & & $(.0000)$ & $(.0000)$ & $(.0000)$ & $(.0000)$ \\
\hline $\begin{array}{l}\text { Coeficiente de } \\
\text { determinación }\end{array}$ & $R^{2}$ & 0.46 & $0.54 \mathrm{~s}$ & 0.41 & 0.36 \\
\hline Estadístico F & $F$ & 88.8 & 80.3 & 43.6 & 32.6 \\
\hline $\begin{array}{l}\text { Estadístico } \\
\text { Durbin-Watson }\end{array}$ & DW & 0.27 & 0.39 & 0.34 & 0.54 \\
\hline
\end{tabular}

Nota: ${ }^{\star \star \star}$ Es al $1 \%,{ }^{\star \star}$ Es al $5 \%$ y ${ }^{\star}$ Es al $10 \%$.

Fuente: elaboración propia con datos de la ENOE. Los valores de probabilidad están entre paréntesis.

aumento en la variable $T A$ de $10 \%$ significa un aumento de $.05 \%, .03 \%$, $.04 \%, .03 \%$ y .01, respectivamente. La variable $T A$ es ES al $5 \%$ en la diferencia $90-70$ y un aumento de $10 \%$ de esta variable se refleja en un aumento de $.01 \%$ en las diferencias del ingreso.

La variable TIL2 es ES a través de todas las diferencias y tiene signo negativo con la excepción de la diferencia 60-70. Un aumento en esta variable se traduce en una reducción de las diferencias del ingreso salarial. En el caso de la diferencia 10-70 la reducción es de .09\% siendo este el valor más alto, mientras que en la diferencia 60-70 la reducción es de .02\% el cual sería el valor más bajo. Adicionalmente, se observa que los resultados del estadístico J tienen un poder de validación eficiente de los instrumentos utilizados.

Donde cada entrada en los cuadros 4 y 5 se refiere al coeficiente para cada variable de la regresión entre cada brecha del ingreso salarial con respecto al séptimo decil por estado y año. El método de estimación es el de GMM usando datos de la ENOE. 
Tomás Gómez, Humberto Ríos y Adriana Zambrano

Cuadro 5. Impacto del salario mínimo en los diferenciales del ingreso, usando el modelo GMM, para los deciles 5-9

\begin{tabular}{|c|c|c|c|c|c|}
\hline Variable & Parámetro & Diferencia 50-70 & Diferencia 60-70 & Diferencia 80-70 & Diferencia $90-70$ \\
\hline \multirow[t]{2}{*}{ Wo } & $\beta_{1}$ & $0.153314^{\star \star \star}$ & $0.096911^{\star \star \star}$ & -0.030353 & -0.043108 \\
\hline & & $(.0000)$ & $(.0000)$ & $(.2780)$ & $(.3683)$ \\
\hline \multirow[t]{2}{*}{ W02 } & $\beta_{2}$ & -0.005979 & 0.025563 & 0.010338 & $0.107532^{\star \star}$ \\
\hline & & $(.8300)$ & $(.2080)$ & $(.6708)$ & $(.0103)$ \\
\hline \multirow[t]{2}{*}{ HW } & $\beta_{3}$ & $-0.006389 * \star *$ & $-0.004028^{\star \star \star}$ & 0.002089 & 0.003432 \\
\hline & & $(0.0002)$ & $(0.0015)$ & $(.1656)$ & (.1835) \\
\hline \multirow[t]{2}{*}{ TA } & $\beta_{4}$ & $0.003088^{* \star \star}$ & $0.001645^{\star \star \star}$ & 0.000241 & $0.001697^{\star \star}$ \\
\hline & & $(.0000)$ & $(.0002)$ & $(.6477)$ & $(.0609)$ \\
\hline \multirow[t]{2}{*}{ TIL2 } & $\beta_{5}$ & $-0.001144^{\star \star}$ & -0.000478 & $0.001054^{\star \star \star}$ & $0.001783^{\star \star \star}$ \\
\hline & & $(.0112)$ & (.1440) & $(.0075)$ & (.0082) \\
\hline $\begin{array}{l}\text { Coeficiente de } \\
\text { determinación }\end{array}$ & $R^{2}$ & .29 & .15 & -.02 & -.03 \\
\hline Estadístico F & $F$ & 18.97 & 5.87 & 4.7 & 14.42 \\
\hline $\begin{array}{l}\text { Estadístico } \\
\text { Durbin-Watson }\end{array}$ & DW & 1.69 & 1.62 & 1.9 & 1.78 \\
\hline
\end{tabular}

Nota: ${ }^{\star \star \star}$ Es al $1 \%$, ${ }^{\star \star}$ Es al $5 \%$ y Es al $10 \%$.

Fuente: elaboración propia con datos de la ENOE. Los valores de probabilidad están entre paréntesis.

De acuerdo con las pruebas de robustez planteadas, se tiene que para las diferencias 80-70 y 90-70 los coeficientes de determinación son cercanos a cero lo que da validez a los resultados. La segunda prueba de robustez son las variables de control las cuales cuentan con el signo positivo para la variable $T A$ que es el esperado y el signo negativo de la variable $H W$ que de igual manera es el signo esperado, de acuerdo con los resultados obtenidos en la estimación que emplea GMM.

En los cuadros 4 y 5 , se puede observar que los coeficientes de correlación para la variable $W O$ disminuyen en relación con los coeficientes obtenidos de la misma variable en los cuadros 2 y 3 . Esto se debe al problema de correlación espuria positiva, problema al que hace referencia Autor et al. (2016) y que se corrige al estimar el modelo con el método GMM, los resultados que se muestran en el cuadro 3 nos muestran coeficientes con valores menores y en el caso de las diferencias 80-70 y 90-70 los coeficientes no son ES. 
Los resultados de la estimación GMM validan los resultados de la estimación con el método de MCO con efectos fijos, esto es que se encuentra una relación positiva entre el salario mínimo y las diferencias del ingreso salarial. Así también, se valida la evidencia que sugiere la existencia de una relación negativa entre empleo en el sector informal y las diferencias del ingreso salarial para el caso de México.

\section{CONCLUSIONES}

Uno de los principales resultados obtenidos, producto de la estimación econométrica, sugiere que el salario mínimo afecta de forma directa a las diferencias del ingreso salarial incrementándolas. En otras palabras, se encuentra que un aumento en el salario efectivo significa un aumento de la desigualdad del ingreso. Los resultados sugieren que el efecto positivo es más fuerte para los tres primeros deciles de la distribución. En otros estudios para países en desarrollo, en su mayoría de América Latina -como son los trabajos de Maloney y Nuñez (2000) y Neumark et al. (2006)-, se tiene que el efecto del salario mínimo es mayor en el fondo de la distribución. Por otro lado, no se encuentra evidencia a favor de la hipótesis de una relación negativa entre salario mínimo y las diferencias del ingreso salarial. Los resultados están en línea con los trabajos de Bosch y Manacorda (2010) y Autor et al. (2016), en ambos trabajos se obtiene que el salario mínimo aumenta las diferencias del ingreso, el primero para el caso de México y el segundo para el caso de Estados Unidos de América. Mientras que en el trabajo de Lin y Yun (2016) encuentran una disminución de las diferencias del ingreso cuando aumenta el salario mínimo para el caso de China. Se empleó un Modelo de Censura para identificar el efecto del salario mínimo sobre las diferencias del ingreso. Además, se utilizaron microdatos de ingreso salarial, los cuales provienen de la ENOE para construir una base de datos a nivel estatal. Se realizaron dos estimaciones econométricas: la primera a través del método de MCo con efectos fijos y la segunda se realizó como prueba de robustez empleando el GMM. Se utilizaron dos variables de control la tasa de asalariados y las horas trabajadas. En cuanto a la tasa de asalariados se encontró que aumenta el diferencial del ingreso a través de todas las diferencias y tiene el signo esperado. El número de horas trabajadas es un indicador de la cantidad de trabajo realizado, se obtiene que es estadísticamente significativo en todas las diferencias salvo en las diferencias 80-70 y 90-70; su efecto es el de disminuir las diferencias del ingreso y también tiene el signo esperado. 
Por otro lado, los resultados sugieren que el tamaño del sector informal tiene un efecto reductor en las diferencias del ingreso salarial. Es decir, los resultados sugieren una disminución de la desigualdad del ingreso cuando aumenta el tamaño del sector informal, efecto que se registra a través de todas las diferencias del ingreso salarial. Estos resultados aportan evidencia la cual soporta la hipótesis de una relación negativa entre empleo informal y diferencias del ingreso salarial. Lo anterior contradice los resultados obtenidos en los trabajos de Zuo (2016), Xue et al. (2014) y Bologna (2016). Los trabajos de Zuo (2016) y Xue et al. (2014) analizan el efecto del sector informal en la desigualdad del ingreso en China encontrando que el sector informal explica una gran parte de la desigualdad del ingreso. De igual manera, en el trabajo de Bologna (2016), en el que se analiza el efecto de la corrupción y el sector informal sobre los niveles de ingreso para el caso de Brasil, se encuentra que el sector informal está asociado con menores niveles de ingreso. Cabe notar que estos resultados están limitados al periodo estudiado y a la muestra considerada en la estimación. Además de que en futuras investigaciones se podrían considerar factores institucionales o bien políticas económicas en conjunto con el efecto del salario mínimo para enriquecer el análisis.

También se encuentra evidencia de que el sector informal podría haber amortiguado los efectos adversos de la crisis financiera global de 2008.

De acuerdo a Gindling (2014) aumentar los salarios mínimos es una herramienta ineficiente para reducir la desigualdad del ingreso. Políticas más eficientes podrían estar orientadas en temas como evitar el incumplimiento de la ley del salario mínimo, mejorar el ingreso en el sector informal, donde los salarios mínimos no aplican incrementar la productividad de largo plazo de trabajadores de familias de bajo ingreso, temas que están abiertos para futuras investigaciones.

\section{BIBLIOGRAFÍA}

Addison, J. y Blackburn, M. (1999), "Minimum wages and poverty", Industrial and Labor Relations Review, vol. 52, núm. 3, DoI <http://dx.doi. org/10.1177/001979399905200302>

Autor, D., Manning, A. y Smith, C. (2016), "The contribution of the minimum wage to US wage inequality over three decades: a reassessment", American Economic Journal: Applied Economics, vol. 8, núm. 1, DOI <http:// dx.doi.org/10.1257/app.20140073> 
Bargain, O. y Kwenda, P. (2010), "Is informality bad? evidence from Brazil, Mexico and South Africa", Institute for the Study of Labor (IZA), IZA Discussion Papers 4711. Recuperado de <ftp.iza.org/dp4711.pdf>

Blunch, N., Canagarajah, S. y Raju, D. (2001), "The informal sector revisited: a synthesis across space and time", Social Protection and Labor Policy and Technical Notes 23308, The World Bank. Recuperado de <https:// www.researchgate.net/publication/237246418_The_Informal_Sector_ Revisited_A_Synthesis_Across_Space_and_Time>

Bologna, J. (2016), "The effect of informal employment and corruption on income levels in Brazil", Journal of Comparative Economics, vol.44, núm. 3, DOI <http://dx.doi.org/10.1016/j.jce.2015.12.001>

Bosch, M. y Manacorda, M. (2010), "Minimum wages and earnings inequality in urban Mexico", American Economic Journal: Applied Economics, vol. 2, núm. 4, DOI <http://dx.doi.org/10.1257/app.2.4.128>

Bouchot, J. A. (2018), The implications of a rise in the minimum wage on the mexican labour market (Tesis Doctoral), Inglaterra, University of Birmingham.

Campos-Vázquez, R. M., Esquivel, G. y Santillán Hernández, A. S. (2018a), "The impact of the minimum wage on income and employment in Mexico", CEPAL Review, núm. 122, DOI <http://dx.doi.org/10.18356/413 e4aea-es>

Campos-Vázquez, R. M., Chavez, E. y Esquivel, G. (2018b), "Estimating top income shares without tax return data: Mexico since the 1990s", Latin American Policy, vol. 9, núm. 1, DOI <http://dx.doi.org/10.1111/lamp. 12143>

Carneiro, F. y Henley, A. (2001), "Modelling formal vs. informal employment and earnings: micro-econometric evidence for Brazil", SSRN Electronic Journal, Working Paper núm. 2001-15, DOI <http://dx.doi.org/10.2139/ ssrn.289826>

Charmes, J. (2000), The contribution of informal sector to GDP in developing countries: assessment, estimates, methods, orientations for the future, Sochi, oecD-eurostat-State Statistical Committee of the Russian Federation. Recuperado de <https://www.researchgate.net/publication/237743723_ The_Contribution_of_Informal_Sector_to_GDP_in_Developing_Countries_Assessment_Estimates_Methods_Orientations_for_the_Future>

Dickens, W. y Lang, K. (1985), "Testing dual labor market theory: a reconsideration of the evidence", National Bureau of Economic Research, Working Paper, núm. 1670, DOI <http://dx.doi.org/10.3386/w1670> 
Duval-Hernández, R., Fields, G. y Jakubson, G. (2016), "Cross-sectional versus panel income approaches: analyzing income distribution changes for the case of Mexico", Review of Income and Wealth, vol. 63, núm. 4, DOI <http://dx.doi.org/10.1111/roiw.12271>

Fields, G. (1975), "Rural-urban migration, urban unemployment and underemployment, and job-search activity in LDC's", Journal of Development Economics, vol. 2, núm. 2, DOI <http://dx.doi.org/10.1016/03043878(75)90014-0>

(2005), "A guide to multisector labour market models", Social Protection Discussion Paper Series, núm. 0505, World Bank, Washington, D.C. Recuperado de <https://www.researchgate.net/publication/37150278_A_ Guide_to_Multisector_Labor_Market_Models>

Funkhouser, E. (1998), "The importance of firm wage differentials in explaining hourly earnings variation in the large-scale sector of Guatemala", Journal of Development Economics, vol. 55, núm. 1, DOI <http://dx.doi. org/10.1016/s0304-3878(97)00058-8>

Gindling, T. (2014), "Does increasing the minimum wage reduce poverty in developing countries?", IZA World of Labor. Recuperado de <https:// wol.iza.org/uploads/articles/30/pdfs/does-increasing-the-minimum-wagereduce-poverty-in-developing-countries.pdf>

Instituto Nacional de Estadística y Geografía (INEGI) (2018), Encuesta Nacional de Ocupación y Empleo (ENOE), población de 15 años y más de edad. Recuperado de <http://www.inegi.org.mx>

Kambayashi, R., Kawaguchi, D. y Yamada, K. (2013), "Minimum wage in a deflationary economy: the japanese experience, 1994-2003”, Labour Economics, vol. 24, DOI <http://dx.doi.org/10.1016/j.labeco.2013.09.005>

Lee, D. (1999), "Wage inequality in the United States during the 1980s: rising dispersion or falling minimum wage?", Quarterly Journal of Economics, vol. 114, núm. 3, DOI <http://dx.doi.org/10.1162/003355399556197>

Lin, C. y Yun, M. S. (2016), "The effects of the minimum wage on earnings inequality: evidence from China”, Income Inequality Around the World, vol. 44, DOI <http://dx.doi.org/10.1108/s0147-912120160000044012>

Maloney, W. (1999), "Does informality imply segmentation in urban labor markets? evidence from sectoral transitions in Mexico", World Bank Economic Review, vol. 13, núm. 2, DOI <http://dx.doi.org/10.1093/wber/ 13.2.275>

Maloney, W. y Nuñez, J. (2000), "Measuring the impact of minimum wages: evidence from Latin America", Policy Research Working Papers, DOI <http:// dx.doi.org/10.1596/1813-9450-2597> 
Moreno-Brid, J. C., Garry, S. y Monroy-Gómez-Franco, L. A. (2014), “El salario mínimo en México", Economía UNAM, vol. 11, núm. 33, DoI <http://dx.doi.org/10.1016/s1665-952x(14)72182-6>

Neumark, D., Schweitzer, M. y Wascher, W. (2005), "The effects of minimum wages on the distribution of family incomes: a nonparametric analysis", Journal of Human Resources, vol. 40, núm. 4, DOI <http://dx.doi. org/10.3368/jhr.xl.4.867>

Neumark, D., Cunningham, W. y Siga, L. (2006), "The effects of the minimum wage in Brazil on the distribution of family incomes: 1996-2001", Journal of Development Economics, vol. 80, núm. 1, DOI <http://dx.doi. org/10.1016/j.jdeveco.2005.02.001>

OIт (2017), "Informe mundial sobre salarios 2016/2017: desigualdades salariales en el lugar de trabajo", Ginebra, Organización Internacional del Trabajo. Recuperado de <https://www.ilo.org/global/publications/books/ WCMS_541632/lang--es/index.htm>

Rand, J. y Torm, N. (2012), "The informal sector wage gap among Vietnamese micro-firms", Journal of the Asia Pacific Economy, vol. 17, núm. 4, DOI <http://dx.doi.org/10.1080/13547860.2012.724544>

Samaniego, N. (2006), "Evolución reciente del mercado de trabajo en México", Estudios sobre las políticas laborales en México, Washington D.C., Banco Interamericano de Desarrollo.

Staneva, A. y Arabsheibani, R. (2014), "Is there an informal employment wage premium? Evidence from Tajikistan", IZA Journal of Labour and Development, vol. 3, núm. 1, DOI <http://dx.doi.org/10.1186/2193-9020-3-1>

Tansel, A. y Kan, E. (2016), "The formal/informal employment earnings gap: evidence from Turkey”, en J. A. Bishop y J. G. Rodríguez (eds.), Inequality after the 20th century: papers from the sixth ECINEQ meeting, Reino Unido, Emerald Group Publishing Limited.

Wu, Y. y Li, T. (2006), "Employment situation and trends in China", en F. Cai y B. Gu (eds.), Green Book of Population and Labor, China, Social Sciences Academic Press.

Xue, J., Gao, W. y Guo, L. (2014), "Informal employment and its effect on the income distribution in urban China", China Economic Review, vol. 31, DOI <http://dx.doi.org/10.1016/j.chieco.2014.07.012>

Zuo, H. (2013), "The earnings differential between formal and informal employees in urban China", Contemporary Economics, vol. 7, núm. 3, DOI <http://dx.doi.org/10.5709/ce.1897-9254.93>

(2016), "How does informal employment impact income inequality?", Applied Economics Letters, vol. 23, núm. 15, DOI <http://dx.doi.org/1 $0.1080 / 13504851.2016 .1142644>$ 
